ITC 2/49

Information Technology and Control

Vol. 49 / No. $2 / 2020$

pp. 317-332

DOI 10.5755/j01.itc.49.2.24072
A Meminductor-based Chaotic System

Received 2019/08/21

Accepted after revision 2019/11/05

HOW TO CITE: Qi, A., Ding, L., Liu, W. (2020). A Meminductor-based Chaotic System. Information Technology and Control, 49(2), 317-332. https://doi.org/10.5755/j01.itc.49.2.24072

\title{
A Meminductor-based Chaotic System
}

\section{Aixue Qi}

College of Automation Engineering;NanJing University of Aeronautics and Astronautics; 29 General Avenue, Jiangning District, Nanjing City, Jiangsu Province, China; phone: +86 135 89424220; e-mail: aixuebz@126.com Faculty of Aerospace Engineering; Binzhou University; No. 391, Yellow River Five Road, Binzhou City, Shandong Province, China; phone: +86 135 89424220; e-mail: aixuebz@126.com

\section{Lei Ding}

Faculty of Aerospace Engineering; Binzhou University; No. 391, Yellow River Five Road, Binzhou City,

Shandong Province, China; phone: +86 150 66995802; e-mail: kiddgamett@163.com

\section{Wenbo Liu}

College of Automation Engineering;NanJing University of Aeronautics and Astronautics; 29 General Avenue, Jiangning District, Nanjing City, Jiangsu Province, China;phone: +86 139 52040018;

e-mail:wenboliu@nuaa.edu.cn

\section{Corresponding author: wenboliu@nuaa.edu.cn}

We propose a meminductor-based chaotic system. Theoretical analysis and numerical simulations reveal complex dynamical behaviors of the proposed meminductor-based chaotic system with five unstable equilibrium points and three different states of chaotic attractors in its phase trajectory with only a single change in circuit parameter. Lyapunov exponents, bifurcation diagrams, and phase portraits are used to investigate its complex chaotic and multi-stability behaviors, including its coexisting chaotic, periodic and point attractors. The proposed meminductor-based chaotic system was implemented using analog integrators, inverters, summers, and multipliers. PSPICE simulation results verified different chaotic characteristics of the proposed circuit with a single change in a resistor value.

KEYWORDS: Chaotic system; meminductor; coexisting attractor; meminductor-based circuit; Dynamic analysis. 


\section{Introduction}

The memristor was theoretically postulated by Professor Leon Chua as the 4th basic circuit element in 1971 [5]. In 2008, successful development of its physical model by researchers in Hewlett Packard labs [24] attracted much attention all over the world $[6,23,4,12,2$, $10,29,19]$. In 2009, the memcapacitor and the meminductor, which extended the memristor, were proposed by Di Ventra et al. [8]. Memristor, meminductor, and memcapacitor are nonlinear circuit elements and can be used to generate chaotic signals $[3,21,27,11,15,22$, $31,26,28]$. Recently, researchers are beginning to realize the potential applications of these nonlinear circuit elements in computer memories, programmable analog circuits, and neural systems.

Compared to memristor-based systems, investigations on meminductor-based chaotic circuits are just at the beginning. The meminductor is not a physical nonlinear circuit element. Therefore, off-the-shelf electronic circuit elements are needed to implement the meminductor. Sah et al. [21] proposed a method to build an expandable architecture of memcapacitor emulator based on memristor emulator. Yang et al. [11] reported a memristor emulator for memristor circuit applications with off-the-shelf solid-state devices. Various features of the memristor emulator are tested via experiments. Liang et al. [15] reported a floating flux-controlled emulator of the meminductor that was implemented without using the memristor. Sanchez-Lopez et al. [22] introduced a new floating memristor emulator circuit based on second-generation current conveyors and passive elements. A mathematical model to characterize the memristor behavior was derived, showing a good accuracy among PSPICE simulations and experimental results. Wang et al. $[31,26]$ put forward a novel meminductor realized by off-the-shelf electronic components and explored its characteristics and equivalent circuit.

In this paper, a meminductor chaotic circuit with only three circuit elements consisting of a linear capacitor, a linear resistor, and a meminductor is proposed. Compared with other meminductor chaotic circuits $[26,28]$, this chaotic system possesses five equilibrium points and three different attractors in its phase trajectory. This system is symmetrical about the original point. It is known that symmetric systems generally possess coexisting attractors $[13,14]$. In Section 2 , the basic model of the meminductor-based chaotic circuit is analyzed. Its equilibrium points, stability, symmetry, and dissipativity are calculated. The dynamical behaviors of this system are analyzed using Lyapunov exponent spectrum and bifurcation. Dynamical behaviors and circuit parameters are reported in Section 3. In Section 4, the numerical experiments are performed to investigate the coexisting bifurcations and coexisting attractors of the system. In Section 5, an appropriate electrical circuit for a simple meminductor chaotic system is designed and implemented in PSPICE. PSPICE simulation results show a good agreement with the theoretical analysis. Finally, conclusion is given.

\section{A Simple Chaotic Circuit with Meminductor}

\subsection{Model of the Meminductor}

Meminductor is a nonlinear circuit element. Defining a flux-controlled model of the meminductor as $\varphi$, the state variables of the meminductor can be described as [4]

$i_{M}=L^{-1}(\rho, \varphi, t) \varphi$
$\dot{\rho}=f(\rho, \varphi, t)$,

$L^{-1}$ is the inverse meminctance, and $\rho$ is the internal state variable of the meminductor and denotes the time-domain integral of the flux linkage $\varphi$. If we define $L^{-1}(\rho, \varphi)=\beta\left(\rho^{2}-1\right), f(\rho, \varphi, t)=-\varphi-d \rho+c \varphi \rho+e \varphi^{2}$, the proposed meminductor model is expressed as:

$$
\left\{\begin{array}{l}
i_{M}=\beta\left(\rho^{2}-1\right) \varphi_{M} \\
\dot{\rho}=-\varphi_{M}-d \rho+c \varphi_{M} \rho+e \varphi_{M}{ }^{2}
\end{array}\right.
$$

where $\beta, d, c$ and $e$ are real constants.

\subsection{Chaotic Circuit Based on Simple Meminductor}

The chaotic circuit with meminductor is shown in Figure 1. It comprises of a linear passive capacitor, 
Figure 1

A chaotic circuit with meminductor

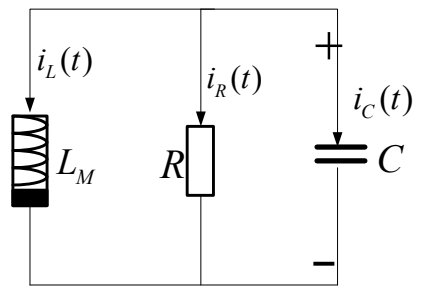

a linear resistor, and a nonlinear flux-controlled meminductor. This electronic circuit can be described by the set of three differential equations given in Eq. (4):

$$
\left\{\begin{array}{l}
\frac{d \varphi_{M}}{d t}=u_{c} \\
c \frac{d u_{c}}{d t}=-\left(i_{L}(t)+\frac{u_{c}}{R}\right) \\
\frac{d \rho}{d t}=-\varphi_{M}+c \varphi_{M} \rho-d \rho+e \varphi_{M}^{2}
\end{array},\right.
$$
Let $\varphi_{M}=x, \quad u_{c}=y, \quad \rho=z, \frac{\beta}{c}=a$ and $\frac{1}{R C}=b$, Eq.
(4) becomes

Figure 2

Meminductor-based chaotic attractors of type I for $e=0.06$

(a) $\mathrm{x}-\mathrm{z}$ phase diagram

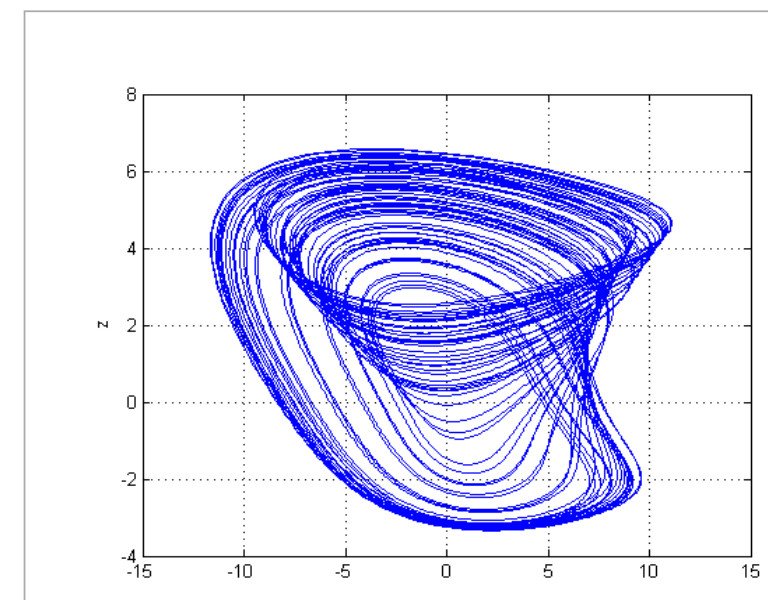

$$
\left\{\begin{array}{l}
\dot{x}=y \\
\dot{y}=-a\left(z^{2}-1\right) x-b y \\
\dot{z}=-x-d z+c x z+e x^{2}
\end{array} .\right.
$$

Assuming $\mathrm{a}=1.1, \mathrm{~b}=0.5, \mathrm{c}=0.06, \mathrm{~d}=0.6$ and initial values in Eq. (5) as (0.1, 0.1, 0.1), the Lyapunov exponents and dimension of each chaotic attractor with different $e$ values can be calculated as shown in Table 1. With different $e$ values, three types of chaotic attractors can be obtained as shown in Figs. 2, 3, and 4. As shown, these chaotic attractors change with different $e$ values.

\section{Table 1}

Lyapunov exponents and Lyapunov dimensions of each chaotic attractor

\begin{tabular}{c|c|c|c}
\hline Type & $e$ & $\begin{array}{c}\text { Lyapunov } \\
\text { exponents }\end{array}$ & $\begin{array}{c}\text { Lyapunov } \\
\text { dimension }\end{array}$ \\
\hline I & 0.06 & $0.2009,0,-1.1575$ & 2.1736 \\
\hline II & 0.6 & $0.1736,0,-1.2349$ & 2.1406 \\
\hline III & 1.2 & $0.12,0,-1.0993$ & 2.1092 \\
\hline
\end{tabular}

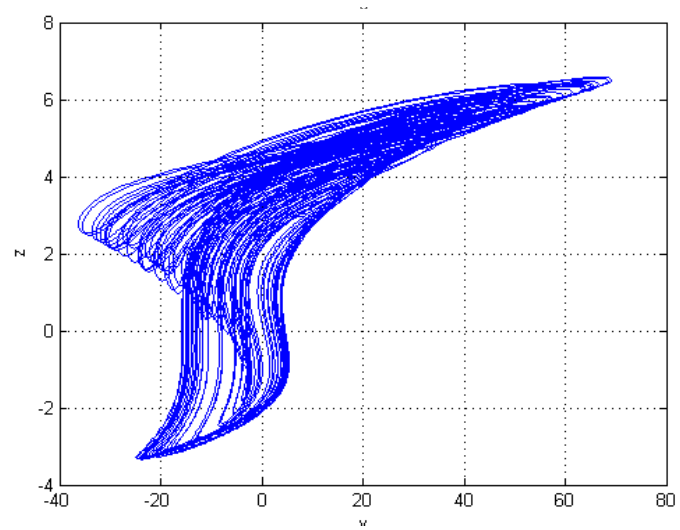

(b) y-z phase diagram 
Figure 3

Meminductor-based chaotic attractors of the type II for e $=0.5$

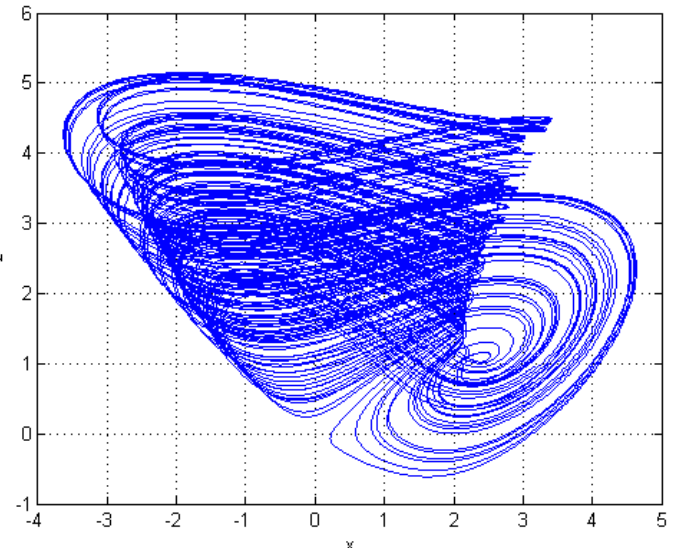

(a) $x-z$ phase diagram

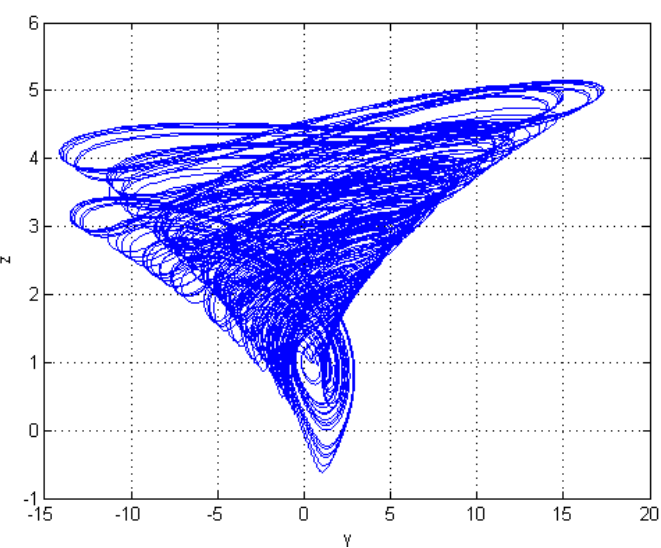

(b) y-z phase diagram

Figure 4

Meminductor-based chaotic attractors of the type III for $e=1$. 2 .

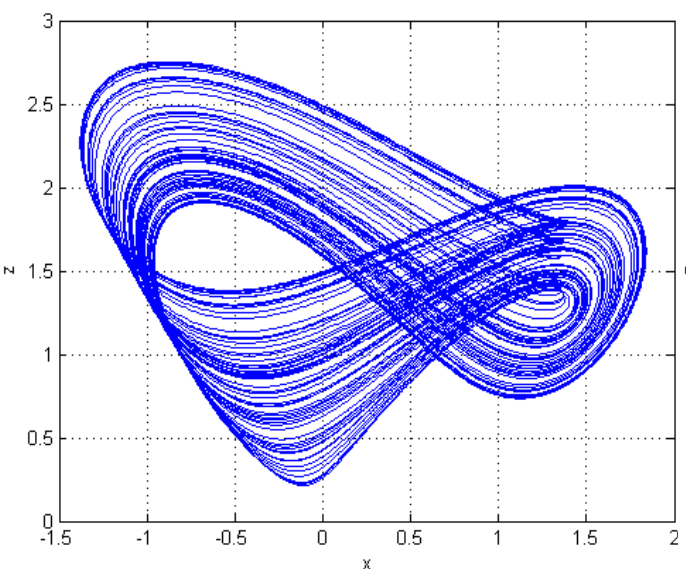

(a) $\mathrm{x}$-z phase diagram

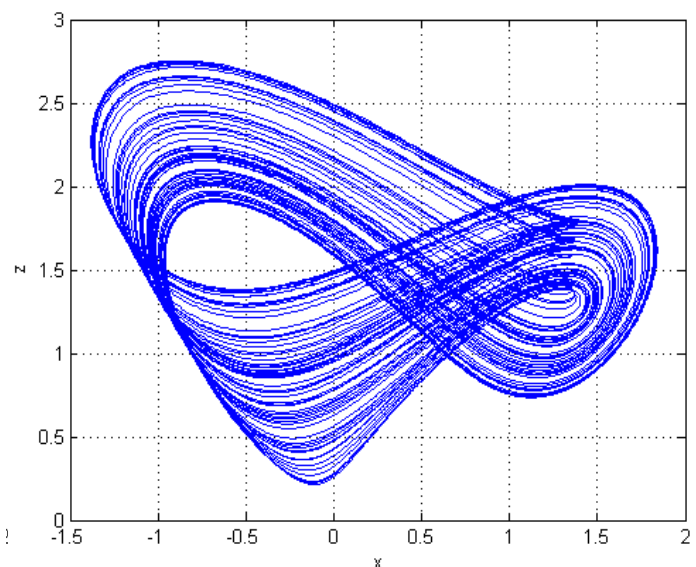

(b) y-z phase diagram

\subsection{Equilibrium Point, Stability, Symmetry and Dissipativity}

The equilibrium of the chaotic system given in Eq. (5) can be calculated by solving $\dot{x}=\dot{y}=\dot{z}=0$. Hence, we obtain five equilibria: one zero equilibrium and four nonzero equilibria given in Eq. (6).

$$
\begin{aligned}
& o_{1}=(0,0,0) ; o_{2}=\left(\frac{1+c+\sqrt{(1+c)^{2}-4 d e}}{2 e}, 0,-1\right) ; \\
& o_{3}=\left(\frac{1+c-\sqrt{(1+c)^{2}-4 d e}}{2 e}, 0,-1\right) ; \\
& o_{4}=\left(\frac{1-c+\sqrt{(1-c)^{2}+4 d e}}{2 e}, 0,1\right) ;
\end{aligned}
$$


$o_{5}=\left(\frac{1-c-\sqrt{(1-c)^{2}+4 d e}}{2 e}, 0,1\right)$.

When $a=1.1, b=0.5, c=0.06, d=0.6, e$ is a variable parameter, the five equilibria are $o_{1}=(0,0,0)$;

$$
\begin{aligned}
& o_{2}=\left(\frac{1.06+\sqrt{1.1236-2.4 e}}{2 e}, 0,-1\right) ; \\
& o_{3}=\left(\frac{1.06-\sqrt{1.1236-2.4 e}}{2 e}, 0,-1\right) ; \\
& o_{4}=\left(\frac{0.94+\sqrt{0.8836+2.4 e}}{2 e}, 0,1\right) ; \\
& o_{5}=\left(\frac{1-c-\sqrt{(1-c)^{2}+4 d e}}{2 e}, 0,1\right) .
\end{aligned}
$$

According to the values of the circuit parameter $e$, there exist three situations as follow:

1 When $e=0.06$, it corresponds to the type I. The equilibria of the system are at $o_{1}=(0,0,0)$; $o_{2}=(17.1,0,-1) ; \quad o_{3}=(2.21,0,-1) ; \quad o_{4}=(16.3,0,1)$; $o_{5}=(-0.73,0,1)$; so the system have five equilibria.

2 When $e=0.6$, it corresponds to the type II. The equilibria of the system are at $o_{1}=(0,0,0)$; $o_{2}=(0.883+0.4687 i, 0,-1) ; o_{3}=(0.883-0.4687 i, 0,-1)$; $o_{4}=(2.053,0,1) ; o_{5}=(-0.487,0,1)$. As we can see, there are two complex equilibria, $o_{2}$ and $o_{3}$, in the system. In the real system, these two complex equilibria are nonexistent, and as such, there are only three equilibria in the system.

3 When $e=1.2$, it corresponds to the type III. The equilibria of the system are at $o_{1}=(0,0,0)$; $o_{2}=(0.44+0.55 i, 0,-1) ; \quad o_{3}=(0.44-0.55 i, 0,-1) ; \quad o_{4}=$ $(1.2,0,1) ; o_{5}=(-0.417,0,1)$, so the system has three equilibria.

In Table 2, the eigenvalues of the Jacobian matrices of type I at different equilibria are calculated. As shown, the eigenvalues of equilibrium $o_{1}$ are all real numbers, two of which are negative, and one is a positive, and the equilibrium point is the saddle point of index 1 . The equilibria $o_{2}, o_{4}$ and $o_{5}$ are unstable saddle-foci nodes of index 1 . The equilibrium $o_{3}$ is unstable saddle-foci nodes of index 2. It is worth noting that the transformation: (x, y, z) $\Leftrightarrow(-\mathrm{x},-\mathrm{y},-\mathrm{z})$ and let system in Eq. (5) be invariant. Correspondingly, if (x,y, z) is a set of solution of system in Eq. (5) for a given set of parameters, then $(-\mathrm{x},-\mathrm{y},-\mathrm{z})$ is also the solution for the same parameters set. It means that the system is symmetrical about the original point. It is known that symmetric systems generally have coexisting attractors $[13,14]$.

Table 2

Eigenvalue of the Jacobian matrices for the equilibria

\begin{tabular}{c|c|c|c|c|c}
\hline$\lambda$ & $O_{1}$ & $O_{2}$ & $O_{3}$ & $O_{4}$ & $O_{5}$ \\
\hline$\lambda_{1}$ & -0.6 & $\begin{array}{c}-1.7706+ \\
2.9984 \mathrm{i}\end{array}$ & $\begin{array}{c}0.1237+ \\
0.8031 \mathrm{i}\end{array}$ & $\begin{array}{c}-1.6403+ \\
2.7354 \mathrm{i}\end{array}$ & $\begin{array}{c}-0.9466+ \\
0.9244 \mathrm{i}\end{array}$ \\
\hline$\lambda_{2}$ & -1.328 & $\begin{array}{c}-1.7706- \\
2.9984 \mathrm{i}\end{array}$ & $0.1237-$ & $-1.6403-$ & $-0.9466-$ \\
\hline$\lambda_{3}$ & 0.346 & 3.4672 & -1.3834 & 3.1585 & 0.7564 \\
\hline
\end{tabular}

To ensure that the system given in Eq. (5) is chaotic, the divergence of Eq.(5) is expressed as $\nabla V=\frac{\partial \dot{x}}{\partial x}+\frac{\partial \dot{y}}{\partial y}+\frac{\partial \dot{z}}{\partial z}=-(b+d)<0$. Because of $b>0, d>0$, the system is dissipative, and the attractor should be chaotic.

\section{Dynamical Behavior and Circuit Parameter}

\subsection{Dynamics with Different Type Chaotic Attractor}

To explore the complex dynamical behaviors of chaotic system of Eq. (5), the Lyapunov exponent spectrum and the bifurcation diagram are calculated. When $\mathrm{a}=$ $1.1, b=0.5, d=0.6$ and $c$ increases from 0 to 1 , with the initial values of $(0.1,0.1,0.1)$ and time step of 0.01 , the Lyapunov exponent spectra of types I, II, and III along with their bifurcation diagrams are shown in Figures. 5,6 , and $\%$. It can be seen that the stable and unstable regions described by these bifurcation diagrams coincided well with those in the Lyapunov exponent spectra.

1 For type I, the chaotic system given in Eq. (5) exhibits a complex dynamical behavior with increasing $c$ values. Different types of chaotic attractors, periodic orbits, and quasi periodic orbit can be generated through numerical simulations as shown in Figure 8. When $c \in[0,0.5] \bigcup[0.72,0.81]$, the system is chaotic since one Lyapunov exponent is $>0$; when $c \in[0.5,0.72]$, the system is periodic since one Lya- 
Figure 5

(a) Lyapunov exponent and (b) bifurcation diagram of type I as a function of parameter $c$

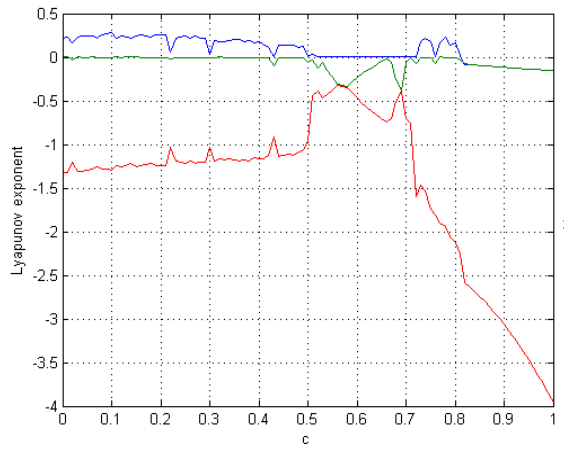

(a) Lyapunov exponent

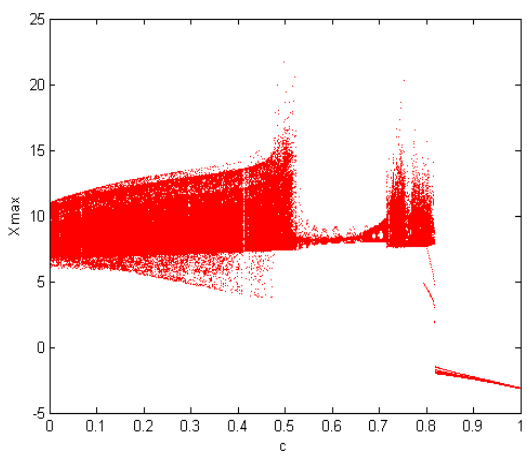

(b) bifurcation diagram

\section{Figure 6}

(a) Lyapunov exponent and (b) bifurcation diagram of type II as a function of parameter $c$

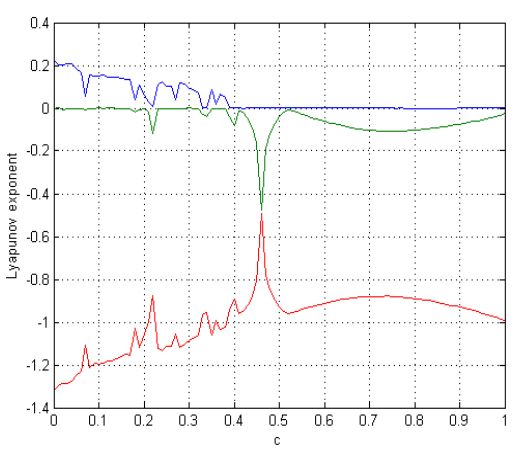

(a) Lyapunov exponent

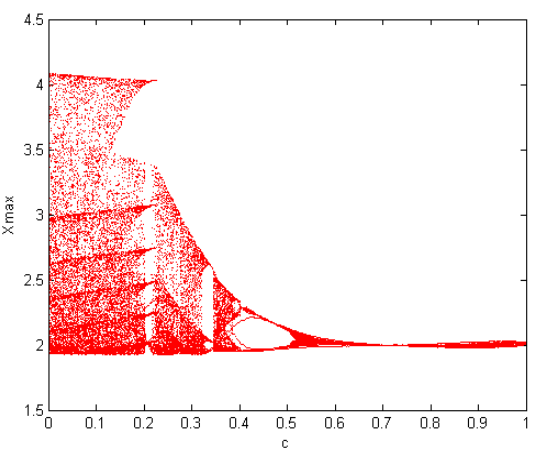

(b) bifurcation diagram

\section{Figure 7}

(a) Lyapunov exponent and (b) bifurcation diagram of type III as a function of parameter $c$

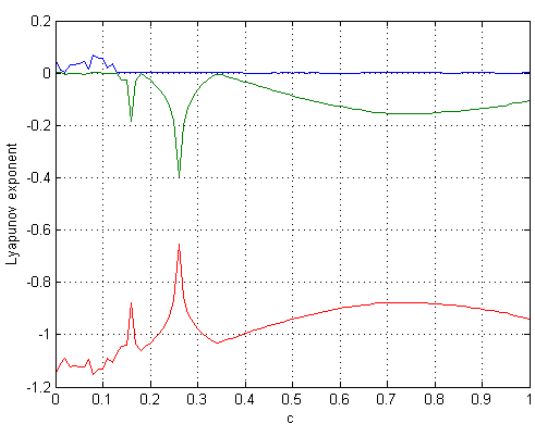

(a) Lyapunov exponent

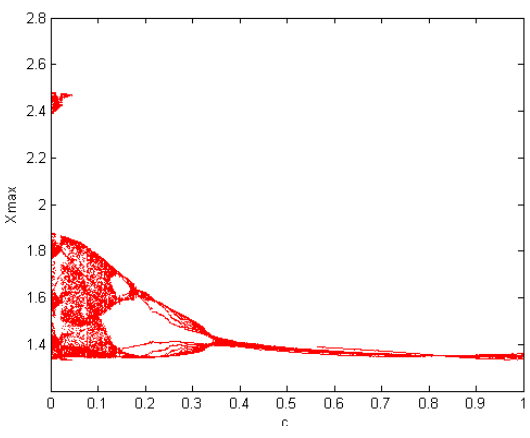

(b) bifurcation diagram 
punov exponent is 0 ; and when $c \in[0.72,1]$, the system is fixed at a point.

2 For type II, when the circuit parameter $\mathrm{c}$ increases, the chaotic system exhibits a complex dynamical behavior when $c \in[0,0.38]$. In addition to existence of two periodic windows $c \in[0.203,0222] \cup[0.33,0.35]$, the system presents a chaotic state in the entire parameter range. For other $c$, the system is periodic.
3 For typeIII, when the circuit parameter $c \in[0,0.13]$, the system is chaotic; when $c \in[018,0.19] \cup$ $[0.34,0.36]$, the system is quasi-periodic since two Lyapunov exponents are 0 . For all other $c$, the system is periodic.

Figure 8(a) shows the phase portraits of type I with different $c$ values to illustrate their chaotic $(c=0.06)$, quasi-periodic $(\mathrm{c}=0.518)$, and periodic behaviors (c $=0.6$ ) while Figure 8(b) and Figure 8(c) illustrate the phase portraits of types II and III.

Figure 8

(a) Phase portraits of the type I (a), type II (b) and type III (c) with different $c$ values

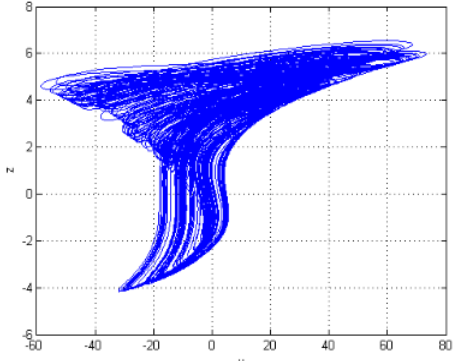

$c=0.06$

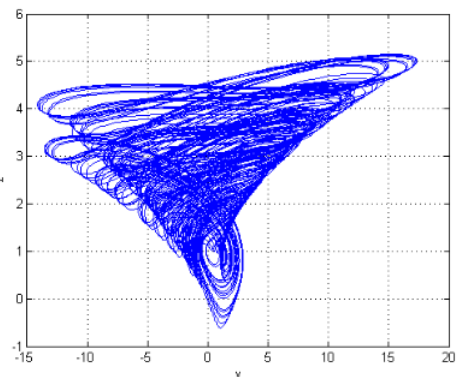

$c=0.06$

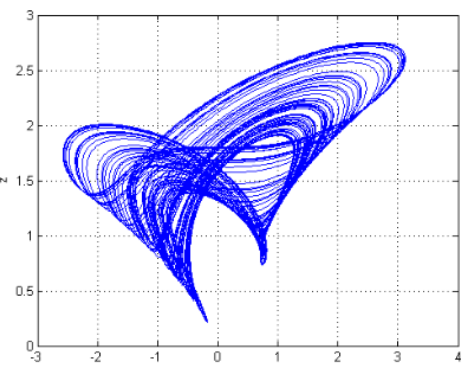

$c=0.06$

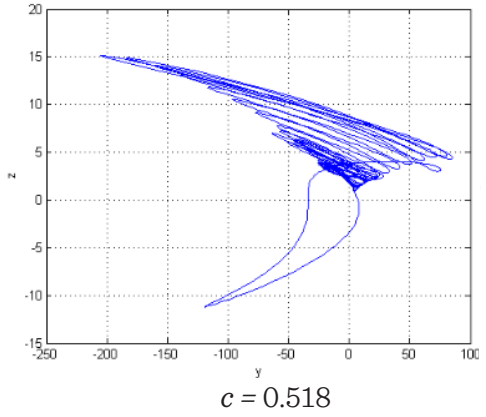

(a) Type I

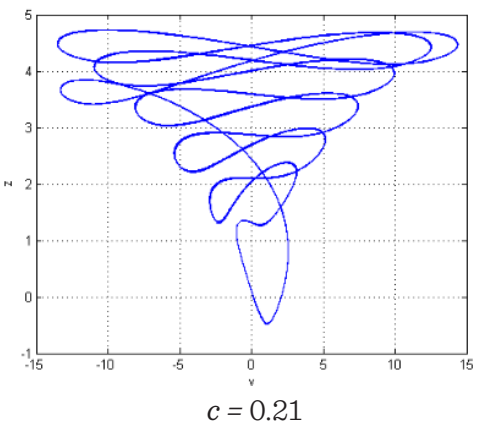

(b) Type II

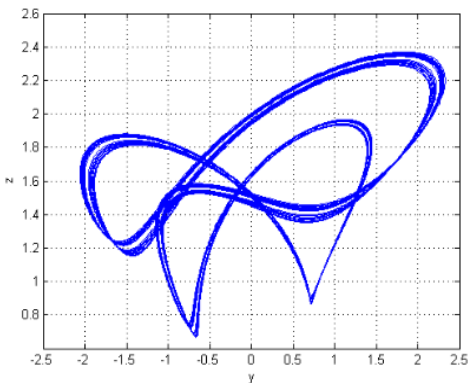

$c=0.35$

(c) Type III
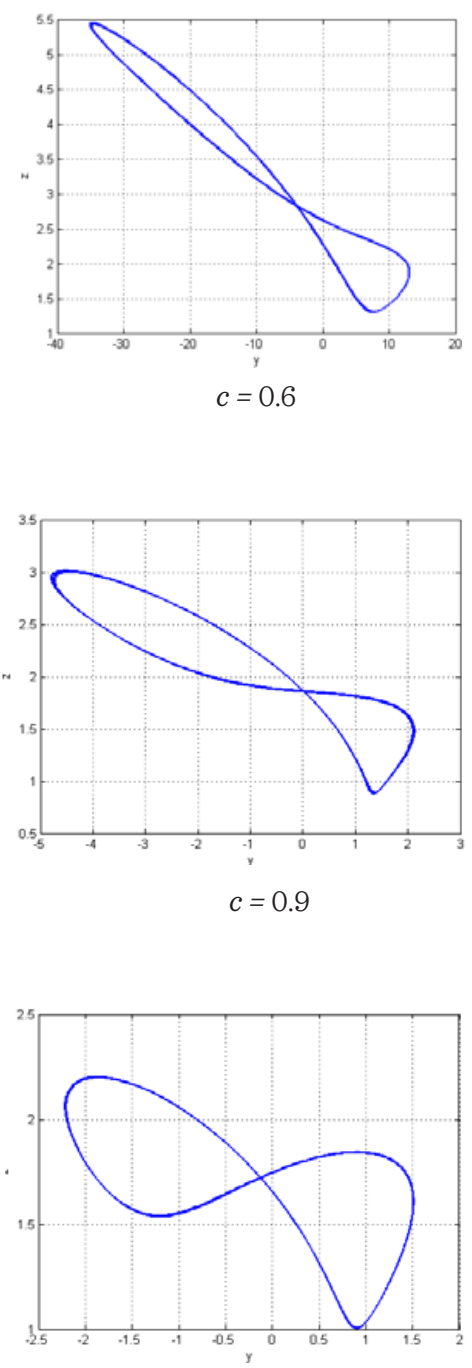

$c=0.7$ 


\subsection{Dynamic Behaviors of System with Parameters E}

Letting $a=0.5, b=1.1, d=0.6, c=0.06$, keeping initial value and the time step the same as mentioned above while varying $e$ from 0 to 1 , the Lyapunov exponent spectrum and the bifurcation diagram are given in Figure 9. With $e=0.001$, the chaotic system in Eq. (5) has

\section{Figure 9}

(a) Lyapunov exponent and (b) bifurcation diagram as a function of parameter $e$

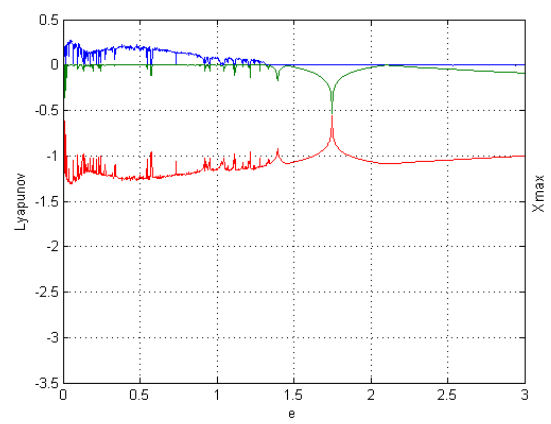

(a) Lyapunov exponent

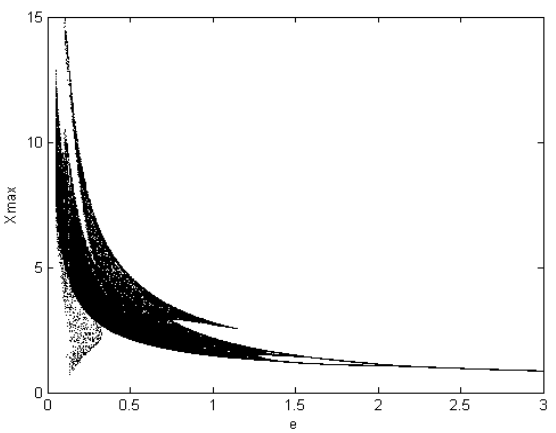

(b) bifurcation diagram

a single periodic orbit in its phase portrait as shown in Figure 10(a). With $e=0.005$, it has two periodic orbits as shown in Figures 10(b). With increasing $e$ values from 0.06 to 1.2 , the phase portraits behave as a chaotic attractor $(e=0.06)$ as shown in Figures 10(c) to $(e)$. As the $e$ value approaches 1.5, the phase portrait becomes quasi periodic orbit as shown in Figures 10(f). At $e=1.7$, the phase portrait has two periodic orbits. At $e=$ 2.3 , the phase portrait becomes a quasi-periodic orbit. Finally, at $e=2.8$, the phase portrait is again a single periodic orbit as shown in Figure 10( $i$ ).

Due to the existence of five unstable equilibria in the system, the dynamic trajectory of the system is very

\section{Figure 10}

Phase portraits on the $x-y$ plane of the chaotic system of Eq. (5) with different $e$ values
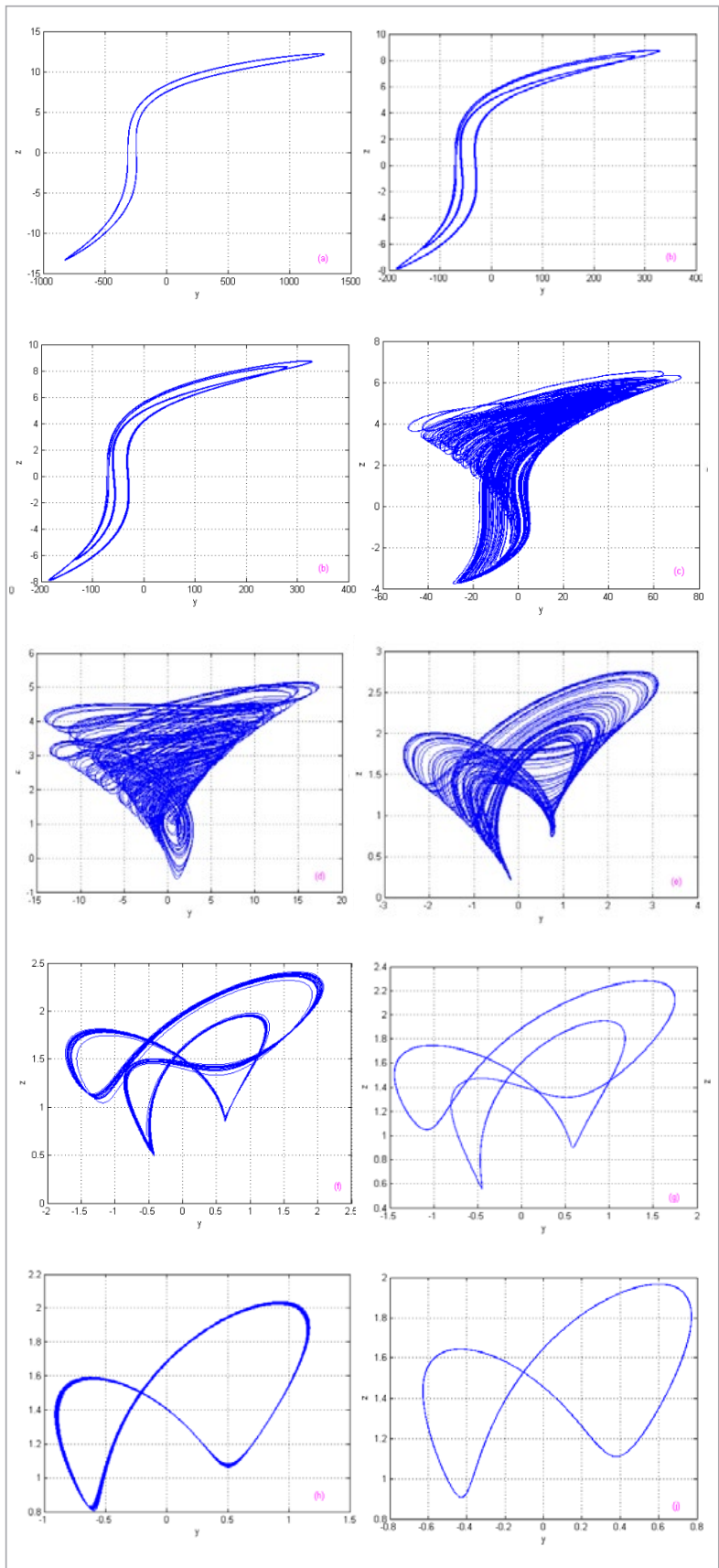

complicated. The bifurcation diagram represents plots of local maxima of the coordinate $\mathrm{x}$ in terms of the control parameter e from 0 to 3 . From the bifurcation diagram in Figure 9 (b), both the steady and unstable regions are covered when $e \in[0.005,1.35]$. 
When all the equilibrium points are unstable, local space of the equilibrium point is scattered and unstable and its phase trajectory generates stretching motion. However, the chaotic system given in Eq. (5) is dissipative and shrinks to zero volume which makes the phase trajectory folded back and generates folding motion. The interaction between the stretching and folding motion leads to chaotic behavior. When system trajectory transits from one unstable region to another, this phenomenon, which produces different types of chaotic attractors, is caused by stretching and folding of system trajectories.

\section{Coexisting Attractor in the System}

In this section, the coexisting periodic and point attractors, coexisting chaotic and point attractors, coexisting periodic attractors, coexisting chaotic attractors of system in Eq. (5) are theoretically and numerically investigated. Let $\mathrm{a}=1.1, \mathrm{~b}=0.5, \mathrm{~d}=0.6, \mathrm{e}=0.06$ while keeping the initial value $\mathrm{x} 0$ at $(3,-0.1,-0.1)$ (blue) and $\mathrm{x} 1$ at $(-3,0.1,-0.1)(\mathrm{red})$ and time step the same as mentioned above and varying $\mathrm{c}$ from 0 to 1 , the Lyapunov exponent spectrum and the coexisting bifurcation diagram are given in Figures 11(a) and (b). As can be

\section{Figure 11}

Circuit parameter $c$ dependence. (a) Lyapunov exponent; (b) bifurcation diagram

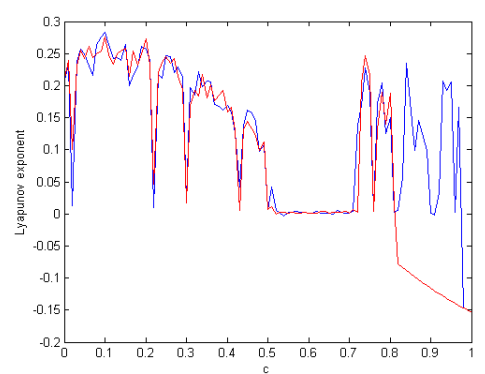

(a) Lyapunov exponent

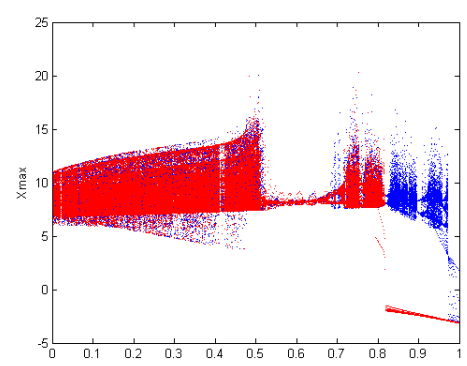

(b) bifurcation diagram seen from Figs.11(a) and (b), the bifurcation diagrams from $\mathrm{x} 0$ and $\mathrm{x} 1$ are very different, there is a reverse period-doubling route to chaos when increasing the value of parameter $\mathrm{c}$ from 0 to 1 .When $\mathrm{c}=0.9$, system in Eq. (5) has periodic and point attractors coexisted as shown in Figure 12 (a). As c decreases to 0.85, system in Eq.

\section{Figure 12}

Coexisting periodic and point attractors of system in Eq. (5) for $c \in[0,1]$ in the $z-x$ and $z$-y planes
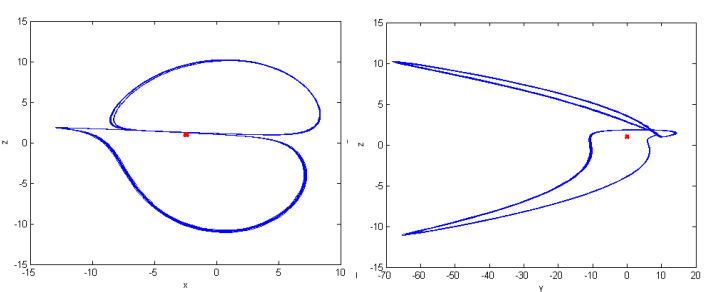

(a) $c=0.9$
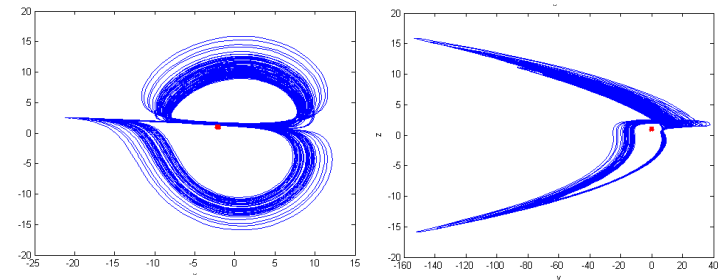

(b) $c=0.85$
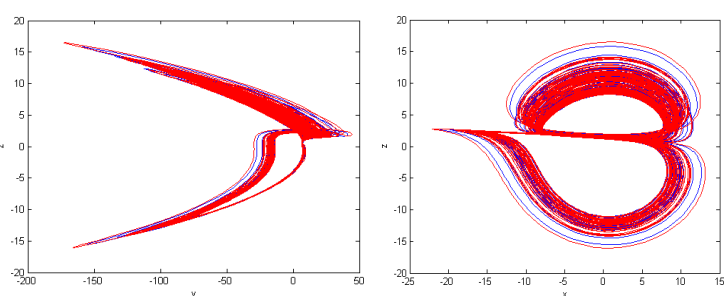

(c) $c=0.8$
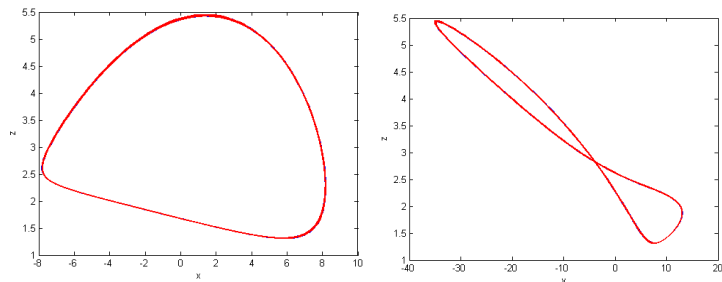

(d) $c=0.55$
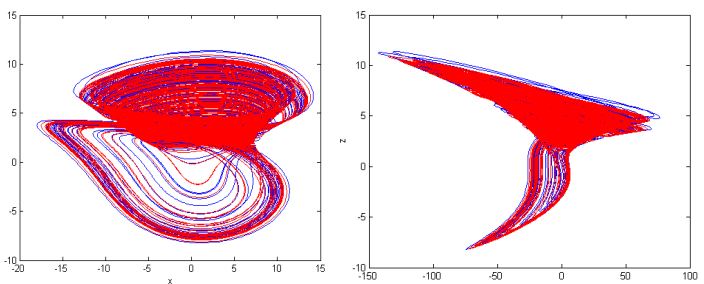

(e) $c=0.4$ 
(5) has chaotic and point attractors coexisted as shown in Figure 12(b). When $\mathrm{c}=0.8$ and c = 0.4, system in Eq. (5) has chaotic attractors coexisted as shown in Figs. 12 (c). For c $=0.55$, system in Eq. (5) has periodic attractors coexisted as shown in Figure 12(d).

\section{Circuit Implementation}

\subsection{The Meminductor Circuit Model}

According to the previously mentioned inverse meminductance equation, its equivalent circuit is implemented as presented in Figure 13. While the basic circuit configuration may seem similar to other proposed circuits [26,33], our meminductor circuit functions differently as its chaotic behaviors can be changed by varying only a single change in one of its circuit element (i.e., a resistor). The circuit comprises of two integrators, inverter, inverting summer, and multipliers. The first integrator U1 gives the output flux $\varphi$. The internal state variable $\rho$ is obtained from the output of the second integrator U2. The output of the U3 is then $i(t)=-\left(\frac{R_{7}}{R_{6}} \rho^{2} \varphi-\frac{R_{7}}{R_{8}} \varphi\right)$. An inverter can be used to achieve $i(t)=\left(\frac{R_{7}}{R_{6}} \rho^{2} \varphi-\frac{R_{7}}{R_{8}} \varphi\right)$, where $R_{6}=R_{8}, \beta=\frac{R_{7}}{R_{6}}$.

To explore the property of the meminductor, a sine voltage of $v_{i}(t)=2 \sin (2 \omega f t)$ is excited at its input. As the frequency of the sine voltage is varied, its $\varphi-i$ curves are shown in Figure14. When $f \leq 1200 \mathrm{~Hz}$, a pinched hysteresis loop is displayed in its $\varphi-i$ plot. By increasing the frequency, the pinched hysteresis loop gradually contracts. When $f>1200 \mathrm{~Hz}$, the pinched hysteresis loop becomes a straight line. With the increase of excitation voltage frequency, the nonlinear function of the meminductor becomes weaker and finally becomes linear. This is similar to the frequency response of the memristor, a common feature of these memory devices. Hence, the characteristics of the meminductor are affected by the excitation frequency. So we can conclude that the proposed meminductor equivalent circuit model satisfies the pinched hysteresis characteristic of the meminductor.

Figure 13

Equivalent circuit of the meminductor model

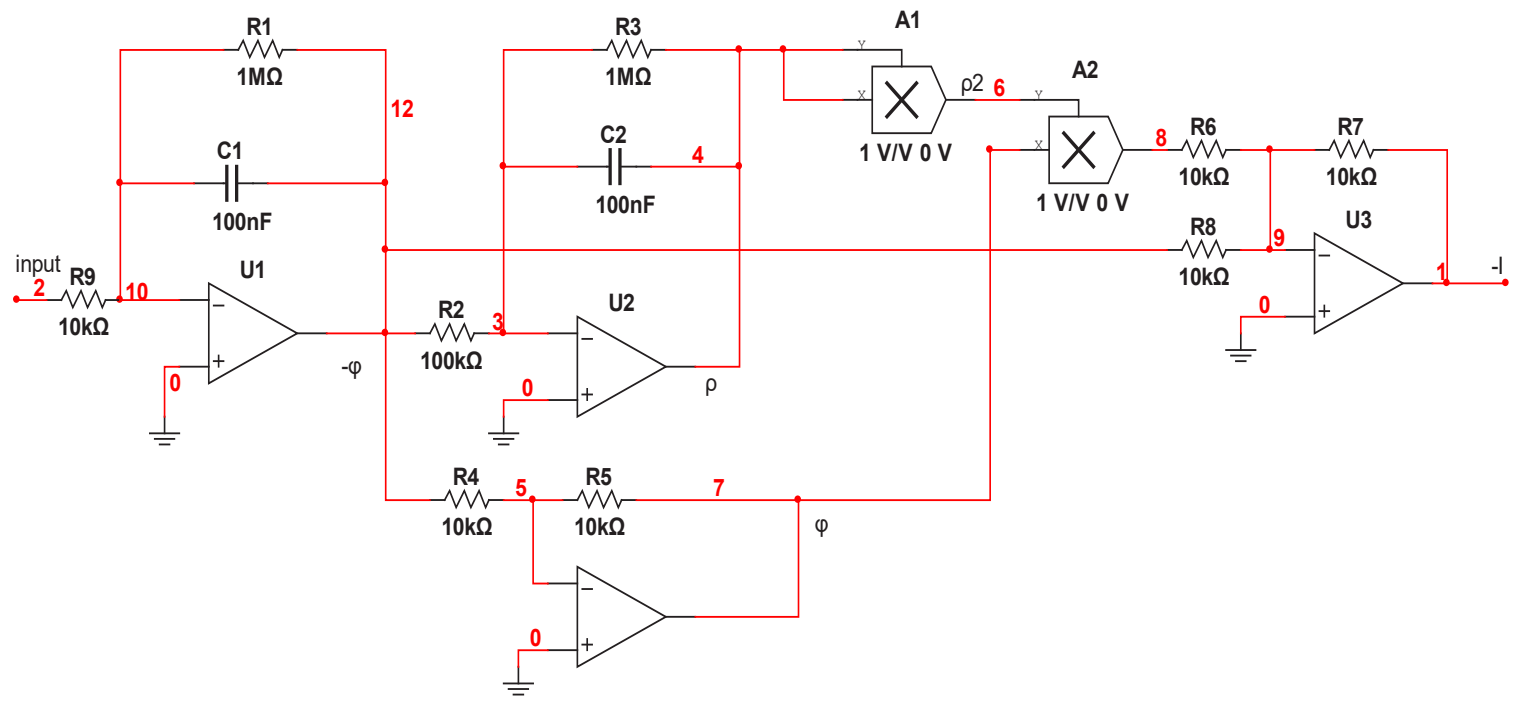


Figure 14

Meminductor $\varphi-i$ curves for $(a) f=200 \mathrm{~Hz},(b) f=400 \mathrm{~Hz},(c) f=800 \mathrm{~Hz}$ and $(d) f=1200 \mathrm{~Hz}$

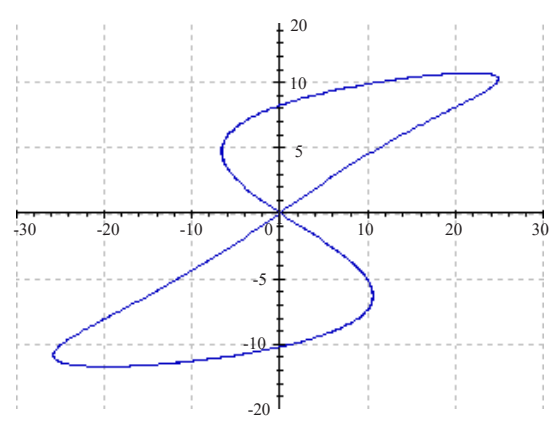

(a) $f=200 \mathrm{~Hz}$

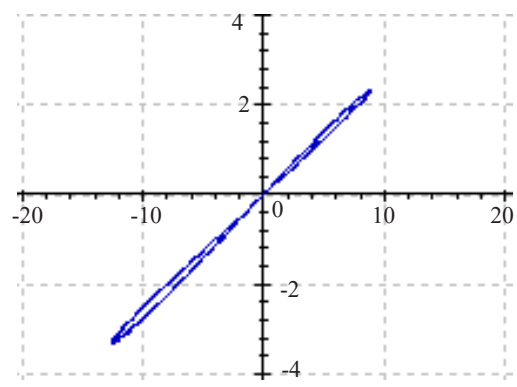

(c) $f=800 \mathrm{~Hz}$

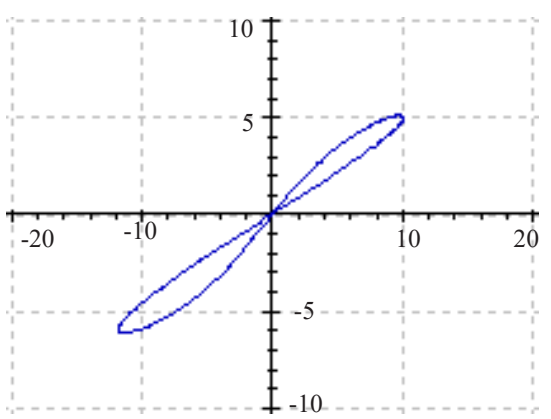

(b) $f=400 H z$

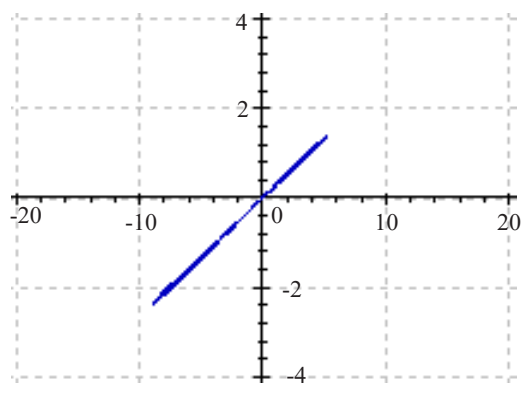

(d) $1200 \mathrm{~Hz}$

\subsection{The Chaotic Circuit Design and Implementation}

Modular design method is applied to obtain a meminductor chaotic circuit and its chaotic attractors are obtained by PSpice simulation. Let $\tau=t / 10 R C,(X, Y$, $Z)=(10 x, 10 y, z)$, Eq. (5) can be transformed to:

$$
\left\{\begin{array}{l}
\dot{X}=\frac{10 R_{1}}{R_{2}} Y \\
\dot{Y}=-\frac{R_{6}}{10 R_{5}} Z^{2} X+\frac{10 R_{6}}{R_{3}} X-\frac{10 R_{6}}{R_{4}} Y \\
\dot{Z}=-\frac{R_{7}}{R_{8}} X-\frac{10 R_{7}}{R_{9}} Z+\frac{R_{7}}{10 R_{10}} X Z+\frac{R_{7}}{100 R_{11}} X^{2}
\end{array}\right.
$$

compared with Eq. (5), $\frac{10 R_{1}}{R_{2}}=1, \frac{R_{6}}{10 R_{5}}=a, \frac{10 R_{6}}{R_{3}}=a$, $\frac{10 R_{6}}{R_{4}}=b, \frac{R_{7}}{R_{8}}=1, \frac{10 R_{7}}{R_{9}}=d, \frac{R_{7}}{10 R_{10}}=c$ and $\frac{R_{7}}{100 R_{11}}=e$, the values of all electronic circuit components are chosen as shown in Figure 15. All amplifiers are of the type 74LF347 whose power supply voltages are $V_{\mathrm{CC}}=12 \mathrm{~V}$ and $V_{\mathrm{EE}}=-12 V . \mathrm{A}_{1}, \mathrm{~A}_{2}, \mathrm{~A}_{3}$ and $\mathrm{A}_{4}$ are analog multipliers of the type AD633. In this circuit, amplifiers are used to realize the basic operation of integration, inversion, and addition, respectively. Adopting an appropriate time scaling, the simulator outputs can directly be visualized on an oscilloscope by feeding the output voltage of $y$ to the $X$ input and the output voltage of $z$ to the $\mathrm{Y}$ input [9].

With all circuit parameters fixed and by changing the value of R11, the different $y-z$ planes are shown in Figure 16. The complex dynamic behaviors of the meminductor chaotic system can be seen by switching from three different trajectories of the system. More precisely, trajectory of the system undergoes a series 
Figure 15

Circuit of meminductor chaotic system
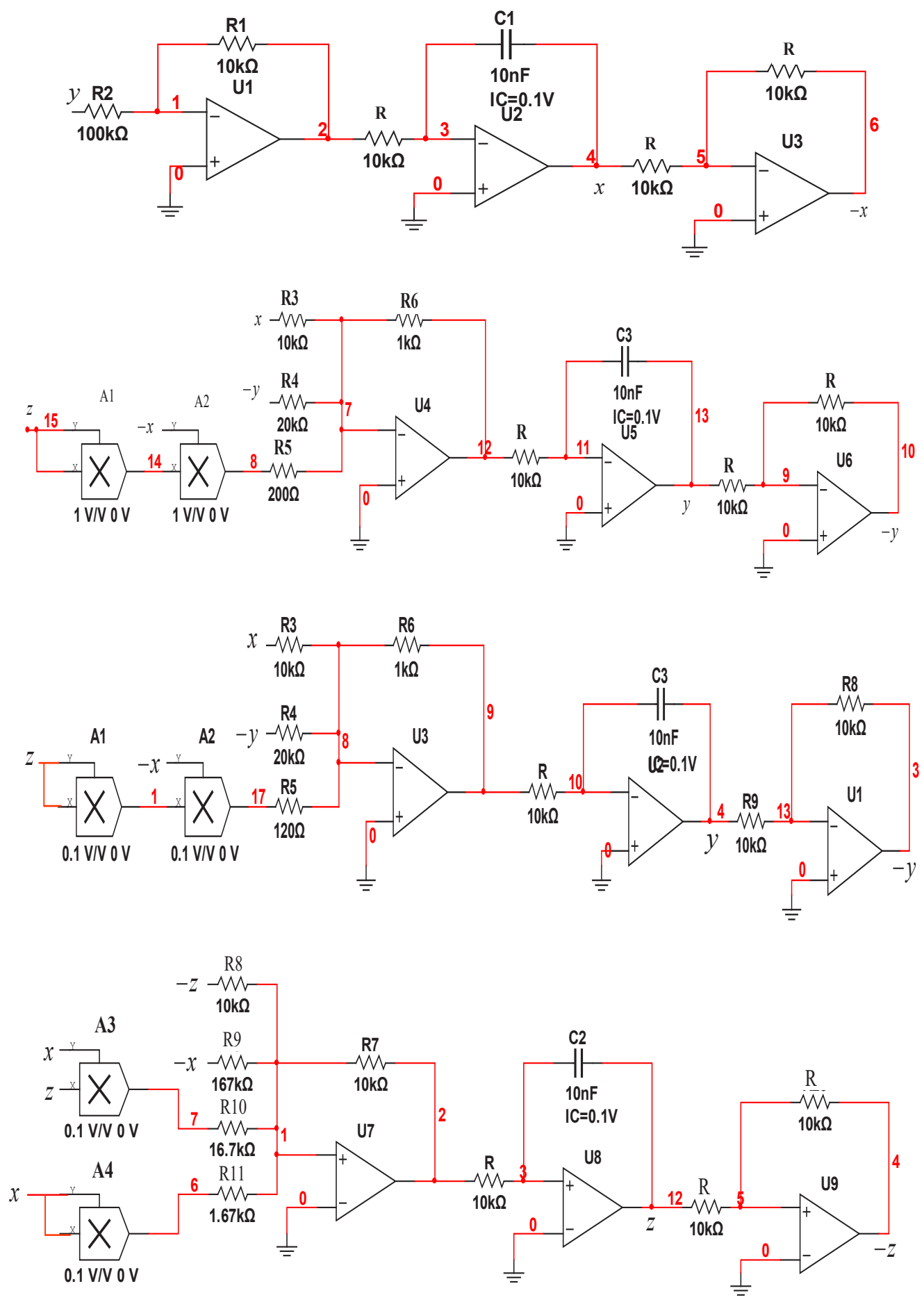


\section{Figure 16}

Chaotic attractors obtained by circuit simulation. ( $a$ ) $R_{11}=30 \mathrm{k} \Omega, y-z$ plane, (b) $R_{11}=18 \mathrm{k} \Omega, y-z$ plane, $(c) R_{11}=1.67 \mathrm{k} \Omega$, $y-z$ plane, time domain waveform of $y($ red $), z($ green $)-t,(d) R_{11}=900 \Omega, y-z$ plane and time domain waveform of $y(r e d)$, $z$ (green)- $t$, (e) $R_{11}=600 \Omega, y-z$ plane and time domain waveform of $y($ red), $z$ (green)- $t$

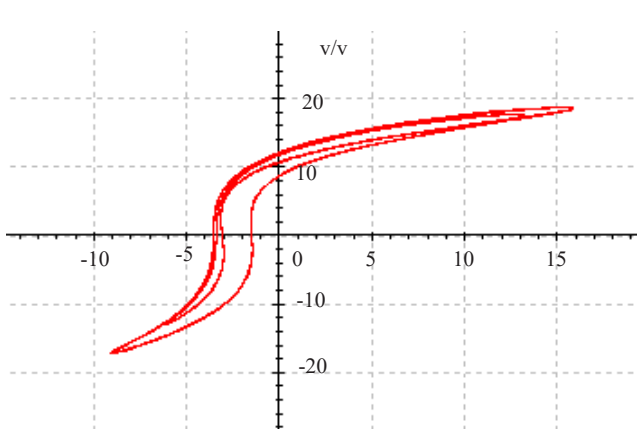

(a)
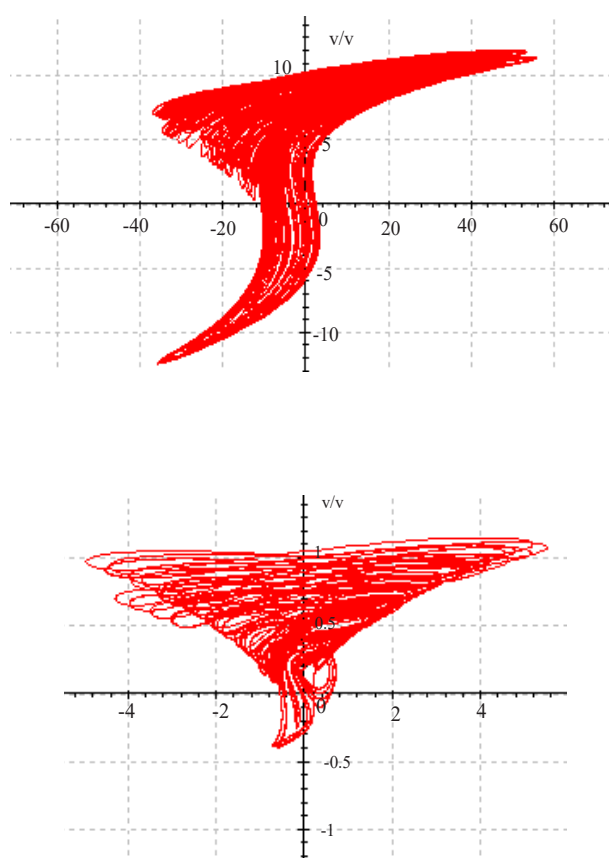

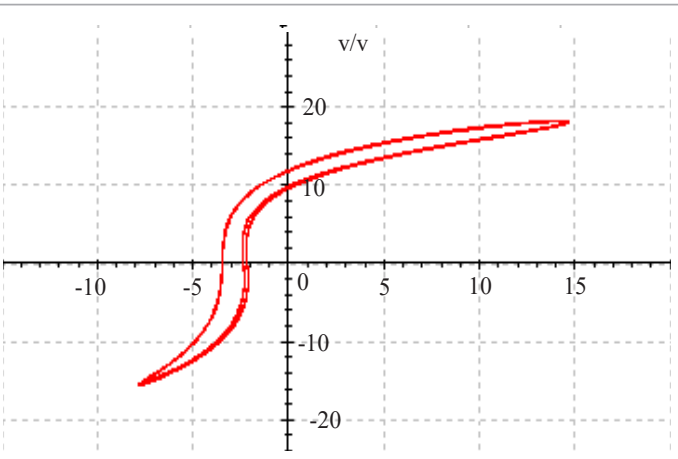

(b)

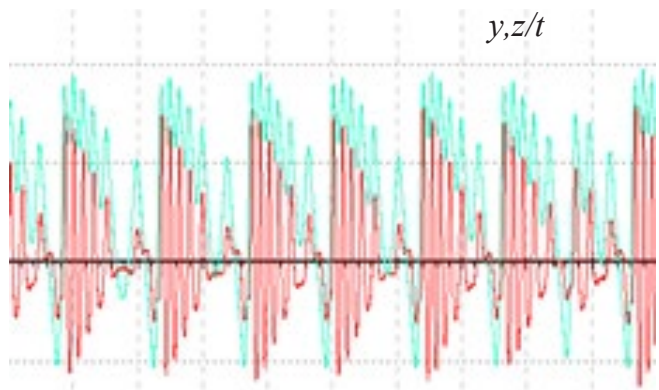

(c)

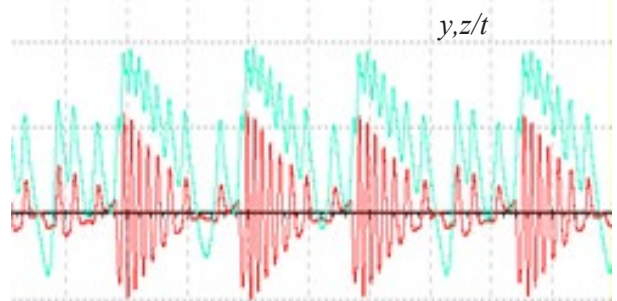

(d)

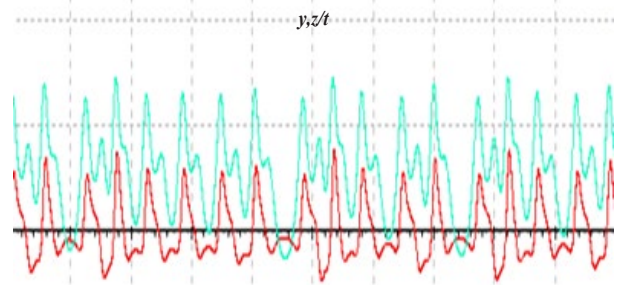

$(e)$ 
of period doubling bifurcation leading to chaos when the control resistor $\mathrm{R} 11$ is decreased from $30 \mathrm{k} \Omega$ to $1.67 \mathrm{k} \Omega$. As shown in Figure 16, the types of chaotic attractors changed when the R11 decreased further. Comparing these phase portraits to those of Matlab simulation shown in Figures 10(a) to (e), the simulation results verify the meminductor-based chaotic circuit. The time domain waveforms of y (red waveform) and $\mathrm{z}$ (green waveform) show that the trajectory of three different types chaotic attractors on the time axis when $R_{11}$ value is changed. This demonstrates that by changing the value of $R_{11}$, different chaotic attractors can be generated.

\section{Conclusions}

We proposed a novel simple chaotic system based on meminductor. Through numerical simulation, it is found that the system exhibits complex dynamic behavior and coexisting attractor. Lyapunov exponent and bifurcation diagrams prove the existence of chaotic attractor. Its phase portraits can be transformed into different chaotic attractors by adjusting

\section{References}

1. Alfa, A. A., Ahmed, K. B., Misra, S., Adewumi, A., Ahuja, R., Ayeni, F., Damasevicius, R. A Comparative Study of Methods for Hiding Large Size Audio File in Smaller Image Carriers. In Emerging Technologies in Computer Engineering: Microservices in Big Data Analytics, 2019, 179-191. Springer Singapore. https://doi. org/10.1007/978-981-13-8300-7_15

2. Bao, B. C., Hu, F. W., Chen, M., Xu, Q., Yu, Y. J. Self-excited and Hidden Attractors Found Simultaneously in a Modified Chua's Circuit. International Journal of Bifurcation and Chaos in Applied Sciences and Engineering, 2015, 25, 1550075. https://doi.org/10.1142/S0218127415500753

3. Biolek, D., Biolek, Z., Biolková, V. PSPICE Modeling of Meminductor. Analog Integrated Circuits and Signal Processing, 2011, 66, 129-137. https://doi.org/10.1007/ s10470-010-9505-5

4. Chen, M., Li, M. Y., Yu, Q., Bao, B. C., Xu, Q., Wang, Q. J. Dynamics of Self-Excited Attractors and Hidden the system parameter e while the initial states and other parameters are held constant. Detailed investigation of the coexisting chaotic attractors, coexisting chaotic and point attractors, coexisting periodic and point attractors, coexisting periodic attractors in the system are theoretically and numerically presented. The meminductor-based chaotic system was implemented using analog electronic components. Simulation results show that the dynamic characteristics of this system are different from the other traditional chaotic systems with distinct dynamic behaviors. The complex chaotic signal generated by the simple meminductor chaotic system can be used in information encryption [20], analysis of physiological signals $[7,18,25]$, communication security $[1,30,16,17,32]$ and other fields.

\section{Acknowledgement}

This research was supported by the Shandong Natural Science Foundation (ZR2014FQ019), Science and Technology Development Planning Project of Binzhou (2014ZC0217) and Binzhou University Research Fund Project (BZXYG1116) and the Key Research and Development Program of Shaanxi Province(No.2018ZDXM-GY-036).
Attractors in Generalized Memristor Based Chua's Circuit. Nonlinear Dynamics, 201, 81, 215-226. https://doi. org/10.1007/s11071-015-1983-7

5. Chua, L. O. Memristor - the Missing Circuit Element. IEEE Transactions on Circuit Theory, 1971, 18, 507519. https://doi.org/10.1109/TCT.1971.1083337

6. Chua, L. O., Kang, S. M. Memristive Devices and Systems. Proceedings of IEEE, 1976, 64, 209-223. https:// doi.org/10.1109/PROC.1976.10092

7. Damasevicius, R., Martisius, I., Jusas, V., Birvinskas, D. Fractional Delay Time Embedding of EEG Signals into High Dimensional Phase Space. Electronics and Electrical Engineering, 2014, 20(8). https://doi.org/10.5755/ j01.eee.20.8.8441

8. Di Ventra, M., Pershin, Y. V., Chua, L. O. Circuit Elements with Memory: Memristors, Memcapacitors, and Meminductors. Proceedings of IEEE, 2009, 97, 17171724. https://doi.org/10.1109/JPROC.2009.2021077 
9. Fan, X., Woźniak, M., Sog, H., Li, W., Li, Y., Shen P. Hळ Control of Network Control System for Singular Plant. Information Technology and Control, 2018, 47(1), 140150. https://doi.org/10.5755/j01.itc.47.1.19982

10. Guoqi, M., Lidan, W., Shukai, D. A Novel Four-Dimensional Memristor Hyperchaotic System with Its Analog Circuit Implementation. Advances in Neural Network, 2015, 157-165. https://doi.org/10.10077/978-3-319-25393-0_18

11. Yang, C. J., Sah, P., Jung, K. S., Cho, S., Kim, H. Memristor Emulator for Memristor Circuit Applications. IEEE Transactions on Circuit Systems, 2012, 59, 2422-2431. https://doi.org/10.1109/TCSI.2012.2188957

12. Yu, Q., Bao, B. C., Xu, Q., Chen, M., Hu, W. Inductorless Chaotic Circuit Based on Active Generalized Memristors. Acta Physica Sinica, 2015, 64, 170503.

13. Kengne, J., Chedjou, J. C., Kom, M., Kyamakaya, K., Kamdoumb Tamba, V. Regular oscillations, chaos, and multistability in a system of two coupled van der Pol oscillators: numerical and experimental studies. Nonlinear Dynamics, 2014, 76, 1119-1132. https://doi. org/10.1007/s11071-013-1195-y

14. Kengne, J., Njitacke, Z. T., Fotsin, H. B. Dynamical Analysis of a Simple Autonomous Jerk System with Multiple Attractors. Nonlinear Dynamics, 2014, 777, 373-386.

15. Liang, Y., Chen, H., Yu, D. S. A Practical Implementation of a Floating Memristor-less Meminductor Emulator. IEEE Transactions on Circuits Systems II, 2014, 61, 299-303. https://doi.org/10.1109/TCSII.2014.2312807

16. Liu, S., Bai, W., Liu, G., Li, W., Srivastava, H. M. Parallel Fractal Compression Method for Big Video Data. Complexity, 2018, 2016976. https://doi. org/10.1155/2018/2016976

17. Liu, S., Pan, Z., Cheng, X. A Novel Fast Fractal Image Compression Method Based on Distance Clustering in High Dimensional Sphere Surface. Fractals, 2017, 25(4), 1740004. https://doi.org/10.1142/S0218348X17400047

18. Martisius, I., Birvinskas, D., Damasevicius, R., Jusas, V. EEG Dataset Reduction and Classification Using Wave Atom Transform. In Artificial Neural Networks and Machine Learning - ICANN 2013, 208-215. Springer. https://doi.org/10.1007/978-3-642-40728-4_26

19. Pershin, Y. V., Di Ventra, M. Emulation of Floating Memcapacitors and Meminductors Using Current Conveyors. Electronics Letters, 2011, 47, 243-244. https://doi.org/10.1049/el.2010.7328

20. Ramasamy, P., Ranganathan, V., Kadry, S., Damaševičius, R., Blažauskas, T. An Image Encryption Scheme
Based on Block Scrambling, Modified Zigzag Transformation and Key Generation Using Enhanced Logistic-Tent Map. Entropy, 2019, 21(7), 656. https://doi. org/10.3390/e21070656

21. Sah, P., Bud, R. K., Yang, C. J., Kim, H. Expandable Circuits of Mutator-Based Memcapacitor Emulator. International Journal of Bifurcation and Chaos in Applied Sciences and Engineering, 2013, 23, 1330017. https:// doi.org/10.1142/S0218127413300176

22. Sánchez-López, C., Mendoza-López, J., Carrasco-Aguilar, M. A., Muñiz-Montero, C. A Floating Analog Memristor Emulator Circuit. IEEE Transactions on Circuit Systems, 2014, 61(5), 309-313. https://doi.org/10.1109/TCSII.2014.2312806

23. Sarmiento-Reyes, A., Hernandez-Martinez, L., Vazquez-Leal, H., Hernandez-Mejal, C., Arango, G. U. D. A Fully Symbolic Homotopy-Based Memristor Model for Applications to Circuit Simulation. Analog Integrated Circuits and Signal Processing, 2015, 85, 65-80. https:// doi.org/10.1007/s10470-015-0579-y

24. Strukov, D. B., Snider, G. S., Stewart, D. R, Stanley, W. R. The Missing Memristor Found. Nature, 2008, 453, 8083. https://doi.org/10.1038/nature06932

25. Timofejeva, I., Poskuviene, K., Cao, M., Ragulskis, M. Synchronization Measure Based on a Geometric Approach to Attractor Embedding Using Finite Observation Windows. Complexity, 2018, 1-16. https://doi. org/10.1155/2018/8259496

26. Wang, G.-Y., Jin, P.-P., Jang X,-W., Shen, Y.-R., Yuan, F., Wang, X.-Y. A Flux-Controlled Model of Meminductor and Its Application in Chaotic Oscillator. Chinese Physics B, 2016, 25(9), 090502. https://doi. org/10.1088/1674-1056/25/9/090502

27. Wang, G. Y., Cai, B. Z., Jin, P. P., Hu, T. L. Memcapacitor Model and Its Application in a Chaotic Oscillator. Chinese Physics, 2016,B(25), 010503. https://doi. org/10.1088/1674-1056/25/1/010503

28. Wang, G. Y., Cui, M. Z., Cai, B. Z., Wang, X. Y., Hu, T. L. A Chaotic Oscillator Based on HP Memristor Model. Mathematical Problems in Engineering, 2015. https://doi. org/10.1155/2015/561901

29. Wang, H., Wang, X., Li, C. D., Chen, L. SPICE Mutator Model for Transforming Memristor into Meminductor. Abstract and Applied Analysis, 2013. https://doi. org/10.1155/2013/281675

30. Wei, W., Woźniak, M., Damasevicius, R., Fan, X., Li, Y. Algorithm Research of Known-Plaintext Attack on Do- 
uble Random Phase Mask Based on WSNs. Journal of Internet Technology, 2019, 20(1), 39-48.

31. Xu, B. R., Wang, G. Y., Shen, Y. R. A Simple Meminductor-Based Chaotic System with Complicated Dynamics. Nonlinear Dynamics, 2017, 88, 2071-2089. https://doi. org/10.1007/s11071-017-3363-y

32. Zheng, P., Shuai, L., Arun, S., Khan, M. Visual Attention Feature (VAF): A Novel Strategy for Visual Tracking
Based on Cloud Platform in Intelligent Surveillance Systems. Journal of Parallel and Distributed Computing, 2018, 120, 182-194. https://doi.org/10.1016/j. jpdc.2018.06.012

33. Zhou, L., Wang, C. H., Zhou, L. L. Generating Hyperchaotic Multi-wing Attractor in a 4D Memristive Circuit. Nonlinear Dynamics, 2016, 85, 2653-2663. https://doi. org/10.1007/s11071-016-2852-8 